\title{
Non-star-forming molecular gas in the Abell 1367 intra-cluster multiphase orphan cloud ${ }^{\star}$
}

\author{
Pavel Jáchym ${ }^{1}$, Ming Sun ${ }^{2}$, Masafumi Yagi ${ }^{3}$, Chong $\mathrm{Ge}^{2}$, Rongxin $\mathrm{Luo}^{2}$, Françoise Combes ${ }^{4}$, Anežka Kabátová ${ }^{1}$, \\ Jeffrey D. P. Kenney ${ }^{5}$, Tom C. Scott ${ }^{6}$, and Elias Brinks ${ }^{7}$
}

\author{
1 Astronomical Institute of the Czech Academy of Sciences, Boční II 1401, 14100 Prague, Czech Republic \\ e-mail: jachym@ig.cas.cz \\ 2 Department of Physics and Astronomy, University of Alabama in Huntsville, Huntsville, AL 35899, USA \\ ${ }^{3}$ National Astronomical Observatory of Japan, 2-21-1, Osawa, Mitaka, Tokyo 181-8588, Japan \\ ${ }^{4}$ Observatoire de Paris, LERMA, Collège de France, CNRS, PSL University, Sorbonne University, 75014 Paris, France \\ 5 Department of Astronomy, Yale University, 46 Hillhouse Avenue, New Haven, CT 06511, USA \\ ${ }^{6}$ Institute of Astrophysics and Space Sciences (IA), Rua das Estrelas, 4150-762 Porto, Portugal \\ 7 Centre for Astrophysics Research, University of Hertfordshire, College Lane, Hatfield AL10 9AB, UK
}

Received 30 November 2021 / Accepted 17 January 2022

\begin{abstract}
We report the detection of $\mathrm{CO}$ emission in the recently discovered multiphase isolated gas cloud in the nearby galaxy cluster Abell 1367. The cloud is located about $800 \mathrm{kpc}$ in projection from the center of the cluster and at a projected distance of $>80 \mathrm{kpc}$ from any galaxy. It is the first and the only known isolated "intra-cluster" cloud detected in X-ray, $\mathrm{H} \alpha$, and CO emission. We found a total of about $2.2 \times 10^{8} M_{\odot}$ of $\mathrm{H}_{2}$ with the IRAM 30-m telescope in two regions, one associated with the peak of $\mathrm{H} \alpha$ emission and another with the peak of X-ray emission surrounded by weak $\mathrm{H} \alpha$ filaments. The velocity of the molecular gas is offset from the underlying $\mathrm{H} \alpha$ emission by $>100 \mathrm{~km} \mathrm{~s}^{-1}$ in the region where the X-ray peaks. The molecular gas may account for about $10 \%$ of the total cloud's mass, which is dominated by the hot X-ray component. The previously measured upper limit on the star formation rate in the cloud indicates that the molecular component is in a non-star-forming state, possibly due to a combination of low density of the gas and the observed level of velocity dispersion. The presence of the three gas phases associated with the cloud suggests that gas phase mixing with the surrounding intra-cluster medium is taking place. The possible origin of the orphan cloud is a late evolutionary stage of a ram pressure stripping event. In contrast, the nearby ram pressure stripped galaxy 2MASX J11443212+2006238 is in an early phase of stripping and we detected about $2.4 \times 10^{9} M_{\odot}$ of $\mathrm{H}_{2}$ in its main body.
\end{abstract}

Key words. galaxies: clusters: individual: Abell 1367 - galaxies: clusters: intracluster medium - galaxies: ISM galaxies: star formation - submillimeter: ISM - galaxies: individual: 2MASX J11443212+2006238

\section{Introduction}

Abell 1367 (A1367) is a nearby $(z=0.022, D=96 \mathrm{Mpc})$ dynamically unrelaxed cluster in the Coma supercluster with $R_{500}=924 \mathrm{kpc}$ and at least two subclusters merging along the southeast-northwest direction (Ge et al. 2019). The cluster's total mass is $\sim 3.3 \times 10^{14} M_{\odot}$ (Boselli \& Gavazzi 2006), which is about one-third of that of the Coma cluster. There are several ram pressure stripped (RPS) galaxies with offset HI distributions and multiphase tails and a prominent infalling group with an associated X-ray and $\mathrm{H} \alpha$ trail caused by ram pressure stripping (e.g., Gavazzi et al. 2001, 2003; Iglesias-Páramo et al. 2002; Scott et al. 2010, 2018; Yagi et al. 2017; Fossati et al. 2019). Recently, an isolated cloud at about $800 \mathrm{kpc}$ in projection from the center of the cluster, not far from the major axis of the cluster, was discovered in narrow-band $\mathrm{H} \alpha$ imaging (Yagi et al. 2017). Due to the absence of an obvious parent galaxy within

* Based on observations carried out under project number 07721 with the Institut de Radio Astronomie Millimétrique (IRAM) 30meter telescope. IRAM is supported by INSU/CNRS (France), MPG (Germany) and IGN (Spain). an $80 \mathrm{kpc}$ search radius, the object was suggested as an orphan cloud (OC). It is projected at the base of a filament of galaxies which extends to the Coma cluster. It is also close to the cluster merger shock. Follow-up XMM observations in the field (Ge et al. 2019) revealed diffuse soft X-ray emission around the same position as the $\mathrm{H} \alpha$ OC. The X-ray OC is asymmetric around its X-ray peak, and a radial surface brightness profile centered on its peak shows an effective radius of $\sim 30 \mathrm{kpc}$. VLT/MUSE IFU observations (Ge et al. 2021) reveal that the $\mathrm{H} \alpha$ OC is elongated $(\sim 10 \mathrm{kpc} \times 20 \mathrm{kpc})$ with a set of narrow filaments connected to the main body. There is a velocity gradient of $\sim 200 \mathrm{~km} \mathrm{~s}^{-1}$ in nearly the east-west direction.

It is likely that the origin of the OC is ISM stripped from an infalling galaxy. Whether it was due to ram pressure stripping, a tidal interaction, or a combination of both, is not clear. Assuming that the speed of the OC to the parent galaxy is less than $\sim 1000 \mathrm{~km} \mathrm{~s}^{-1}$ (cf. the A1367 radial velocity dispersion of $726 \mathrm{~km} \mathrm{~s}^{-1}$ ), the OC should have survived in the cluster environment for $>70$ Myr. The MUSE observations and line diagnostics suggest high, about solar metallicity of the warm ionized gas (Ge et al. 2021), implying a massive parent for the OC. The 

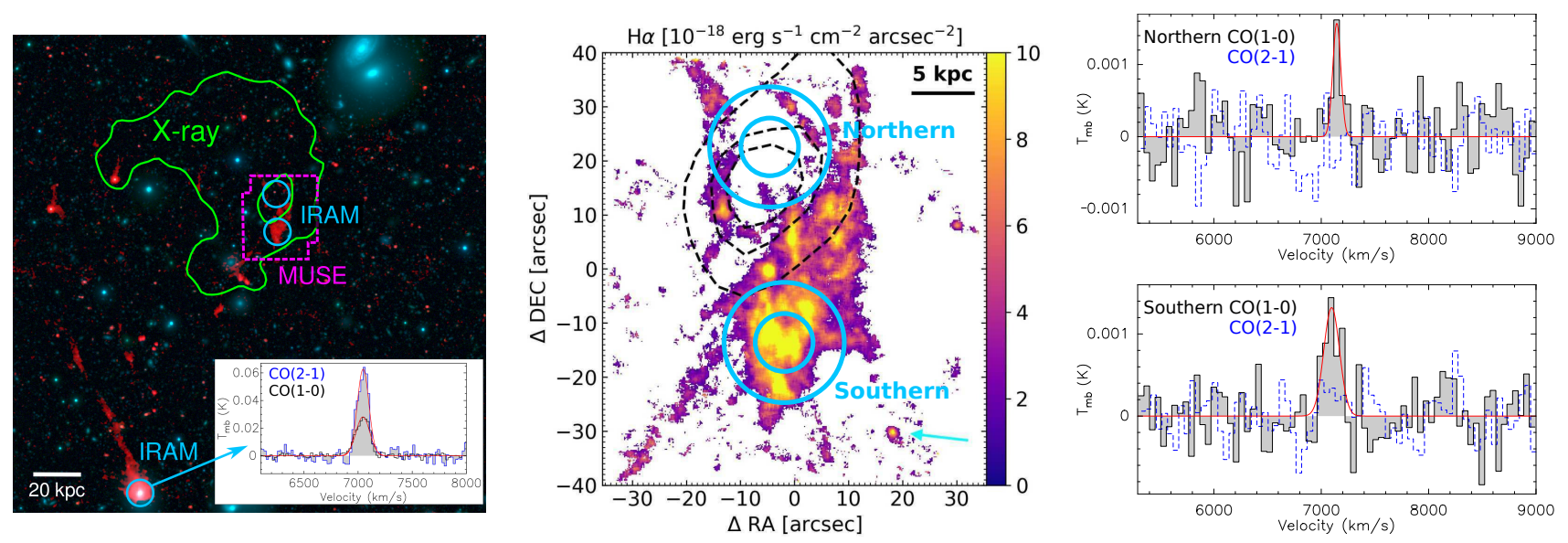

Fig. 1. Multiwavelength observations of the A1367 OC. Left: A1367 OC - the green contour outlines its X-ray morphology (0.5-2 keV XMM observations) overlaid on a Subaru three-color composite image (red, H $\alpha$; green, $r$-band; and blue, $g$-band). The dashed magenta region shows the MUSE FOV, and the blue circles indicate the IRAM CO(1-0) beams. Image adapted from Ge et al. (2021). The galaxy 2MASX J11443212+2006238 with its $85 \mathrm{kpc}$ long $\mathrm{H} \alpha$ ram pressure stripped tail is visible to the south of the OC. The CO spectra from a pointing centered on this galaxy are shown in the inset. Middle: MUSE H $\alpha$ image of the OC (Ge et al. 2021) showing the positions of the CO(1-0) and $\mathrm{CO}(2-1)$ beams (blue circles, HPBW of $21.7^{\prime \prime} \approx 9.7 \mathrm{kpc}$ and $10.9^{\prime \prime} \approx 4.9 \mathrm{kpc}$, respectively). The black dashed contours show the peak X-ray emission of the OC. Right: $\mathrm{CO}(1-0)$ lines detected at the positions over the northern X-ray peak (top panel) and the southern $\mathrm{H} \alpha$ peak (bottom panel). Velocity corresponds to $v_{\mathrm{rad}}=c z /(z+1)$. The corresponding $\mathrm{CO}(2-1)$ spectra divided by a factor of 3 are shown with dotted blue lines. In the southern region (bottom panel), a marginal $\mathrm{CO}(2-1)$ line detection is suggested from a positive signal at velocities around the $\mathrm{CO}(1-0)$ line central velocity.

temperature of the X-ray OC, $1.6 \pm 0.1 \mathrm{keV}$, is higher than typical temperatures of X-ray RPS tails of cluster late-type galaxies $(\sim 0.9 \mathrm{keV}$, Sun et al. 2010), which, together with the large distance to any possible parent galaxy, suggests an advanced evolutionary stage of the OC as it mixes with the surrounding ICM. The bright $\mathrm{H} \alpha$ part of the cloud may be associated with the only surviving cold clouds, while the bulk of the X-ray OC is free of cold gas now. The A1367 OC presents a unique laboratory to study the evolution of the ISM far away from the parent galaxy.

Here we present the first $\mathrm{CO}$ observations of the $\mathrm{H} \alpha$-bright part of the OC. These observations show that the OC also contains a cold molecular gas component, in addition to the warm and hot ionized gas phases. Despite the presence of the molecular gas, only a very small amount of star formation was found in the OC (Ge et al. 2021), indicating that the conversion of the molecular gas to stars is extremely inefficient. The A1367 OC with its multiphase components of cold, warm, and hot gas is unique. In the Virgo cluster, several isolated HI clouds with no optical counterparts are known (Davies et al. 2004; Kent et al. 2007), as well as the low-mass, star-forming $\mathrm{H} \alpha$ cloud SECCO 1 (Beccari et al. 2017). There are also examples of isolated HI clouds in galaxy groups (e.g., Wong et al. 2021).

\section{Observations}

The observations were carried out with the IRAM 30-m telescope operated by the Institut de Radio Astronomie Millimétrique (IRAM) at Pico Veleta, Spain, from 01 June 2021 to 07 June 2021 (PI Jáchym, project ID 077-21). The EMIR receiver in the E090 and E230 bands was used to observe at the frequencies of the ${ }^{12} \mathrm{CO}(1-0)\left(v_{\text {rest }}=115.271 \mathrm{GHz}\right)$ and the ${ }^{12} \mathrm{CO}(2-1)\left(v_{\text {rest }}=230.538 \mathrm{GHz}\right)$ lines simultaneously. The FTS spectrometer, together with the WILMA autocorrelator, were connected to both receivers. Symmetric wobbler switching with the maximum secondary reflector throw of $\pm 60^{\prime \prime}$ and $t_{\text {phase }}=$ $1.5 \mathrm{~Hz}$ was used. Observing conditions varied from excellent with PWV as low as $1-2 \mathrm{~mm}$ to bad with PWV $>10 \mathrm{~mm}$. Two nights (04 and 05 June) were lost due to unstable conditions and high wind.

Two regions of the OC were observed, one ("southern", J2000 RA 11:44:22.89, Dec +20:10:31.5) covering the main peak of the $\mathrm{H} \alpha$ and the other ("northern", J2000 RA 11:44:23.17, Dec +20:11:07.6) covering the neighboring peak of the X-ray emission (see Fig. 1, middle panel). The latter region lacks bright $\mathrm{H} \alpha$ features. These were deep observations, with onsource time for the southern and northern regions of $4.85 \mathrm{~h}$ and $3.8 \mathrm{~h}$, respectively. In addition, we targeted the nearby galaxy 2MASX J11443212+2006238 with a pointed observation of $0.8 \mathrm{~h}$. The half power beamwidth (HPBW) of the IRAM $30 \mathrm{~m}$ main beam is $\sim 21.7^{\prime \prime}$ and $10.9^{\prime \prime}$ at the $\mathrm{CO}(1-0)$ and $\mathrm{CO}(2-1)$ frequencies, respectively. The data were reduced using CLASS from the GILDAS ${ }^{1}$ software package developed at IRAM. The corrected antenna temperatures, $T_{\mathrm{A}}^{*}$, provided by the IRAM $30 \mathrm{~m}$ calibration pipeline were converted to a main-beam brightness temperature by $T_{\mathrm{mb}}=T_{\mathrm{A}}^{*} F_{\mathrm{eff}} / \eta_{\mathrm{mb}}$, using a main beam efficiency of $\eta_{\mathrm{mb}}=0.78$ at $115 \mathrm{GHz}$ and 0.59 at $230 \mathrm{GHz}$, and the forward efficiencies $F_{\text {eff }}$ of 0.94 and 0.92 , respectively. The flux density-to-main beam antenna temperature conversion factor is $\sim 5 \mathrm{Jy}_{\text {beam }}^{-1} \mathrm{~K}^{-1}$ for both bands (see Jáchym et al. 2017).

\section{Results}

We detected $\mathrm{CO}(1-0)$ emission in both the southern and northern regions. Their $\mathrm{CO}(1-0)$ spectra, smoothed to $52 \mathrm{~km} \mathrm{~s}^{-1}$ resolution, are shown in the right panels of Fig. 1. Low rms values of about $0.31 \mathrm{mK}$ and $0.46 \mathrm{mK}$, respectively, were achieved. In the southern region covering the $\mathrm{H} \alpha$ peak, the $\mathrm{CO}(1-0)$ line is detected with an $S / N=T_{\text {peak }} / \sigma_{\text {rms }} \sim 4.4$, and an integrated $S / N=I_{\mathrm{CO}} / \sigma_{\text {int }} \sim 8.7$, where $\sigma_{\text {int }}=$ $F W H M \sigma_{\text {rms }} \sqrt{\Delta v_{\text {ch }} / F W H M}$ corresponds to the noise over the spectral channels (of width $\Delta v_{\text {ch }}$ ) covered by the line. In Fig. 1 (right panel), the $\mathrm{CO}(1-0)$ spectral line is fitted with a Gaussian

1 http://wWw.iram.fr/IRAMFR/GILDAS 
P. Jáchym et al.: Molecular gas in intra-cluster orphan cloud

Table 1. Properties of the CO detections in the OC and the RPS galaxy 2MASX J11443212+2006238.

\begin{tabular}{lcccccccc}
\hline \hline Region & Line & $\begin{array}{c}\sigma_{\mathrm{rms}} \\
(\mathrm{mK})\end{array}$ & $\begin{array}{c}c z /(z+1) \\
\left(\mathrm{km} \mathrm{s}^{-1}\right)\end{array}$ & $\begin{array}{c}F W H M \\
\left(\mathrm{~km} \mathrm{~s}^{-1}\right)\end{array}$ & $\begin{array}{c}T_{\mathrm{mb}}^{\text {peak }} \\
(\mathrm{mK})\end{array}$ & $\begin{array}{c}I_{\mathrm{CO}} \\
\left(\mathrm{K} \mathrm{km} \mathrm{s}^{-1}\right)\end{array}$ & $\begin{array}{c}L_{\mathrm{CO}} \\
\left(10^{7} \mathrm{~K} \mathrm{~km} \mathrm{~s}^{-1} \mathrm{pc}^{2}\right)\end{array}$ & $\begin{array}{c}M_{\mathrm{H}_{2}} \\
\left(10^{8} M_{\odot}\right)\end{array}$ \\
\hline Northern & $\mathrm{CO}(1-0)$ & 0.46 & $7145.1 \pm 14.3$ & $85.6 \pm 30.7$ & 1.55 & $0.14 \pm 0.05$ & $1.7 \pm 0.6$ & 0.8 \\
Southern & $\mathrm{CO}(1-0)$ & 0.31 & $7096.9 \pm 16.1$ & $178.0 \pm 29.2$ & 1.37 & $0.26 \pm 0.04$ & $3.1 \pm 0.5$ & 1.4 \\
2MASX & $\mathrm{CO}(1-0)$ & 1.3 & $7044.1 \pm 1.7$ & $150.3 \pm 4.0$ & 28.2 & $4.5 \pm 0.1$ & $53.7 \pm 1.2$ & 24.2 \\
2MASX & $\mathrm{CO}(2-1)$ & 3.6 & $7046.3 \pm 2.0$ & $123.8 \pm 4.3$ & 61.9 & $8.2 \pm 0.3$ & $24.5 \pm 0.9$ & 13.5 \\
\hline
\end{tabular}

Notes. The rms sensitivity values are given for $52 \mathrm{~km} \mathrm{~s}^{-1}$ channels in the OC regions and $20 \mathrm{~km} \mathrm{~s}^{-1}$ channels in the 2MASX galaxy.

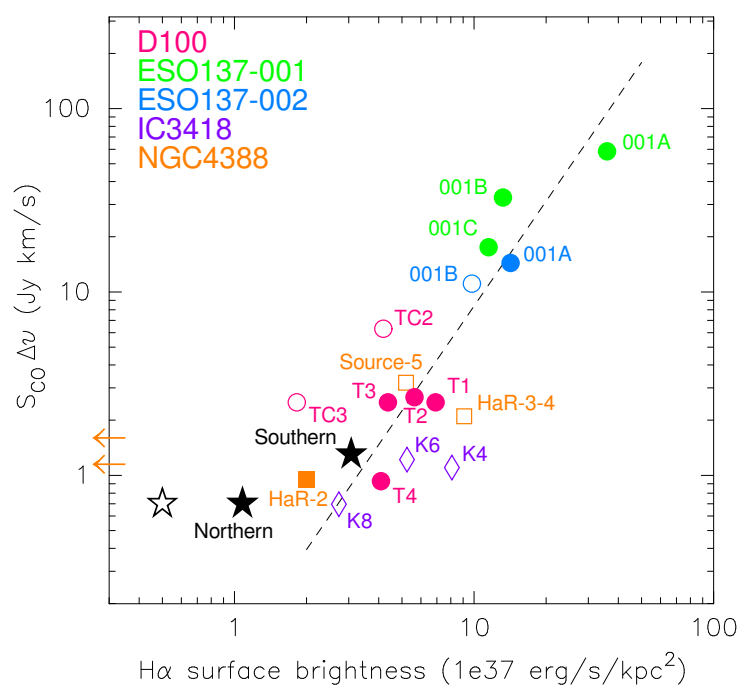

Fig. 2. CO integrated flux vs. $\mathrm{H} \alpha$ surface brightness measured in a sample of ram pressure stripped gas tails (Jáchym et al. 2017, their Fig. 9). The location of the two OC regions is shown with filled stars. The open star symbol corresponds to the northern region surface brightness, when the full area of the $\mathrm{CO}(1-0)$ beam is used instead of the actual $\mathrm{H} \alpha$ emitting area within the beam (filled star).

profile with a central velocity of $\sim 7096 \mathrm{~km} \mathrm{~s}^{-1}$ and a FWHM of $178 \mathrm{~km} \mathrm{~s}^{-1}$ (see also Table 1). The integrated intensity is $I_{\mathrm{CO}}=0.26 \pm 0.04 \mathrm{~K} \mathrm{~km} \mathrm{~s}^{-1}$, which corresponds to a mean molecular gas column density of $\sim 1.2 M_{\odot} \mathrm{pc}^{-2}$ and a molecular gas mass of $\sim 1.4 \times 10^{8} M_{\odot}$, following the standard relation of Solomon \& Vanden Bout (2005), and assuming a Galactic COto- $\mathrm{H}_{2}$ conversion factor of $2 \times 10^{20} \mathrm{~cm}^{-2}\left(\mathrm{~K} \mathrm{~km} \mathrm{~s}^{-1}\right)^{-1}$.

Quite unexpectedly, $\mathrm{CO}(1-0)$ was also detected in the northern region pointed over the neighboring X-ray peak, which is mostly devoid of $\mathrm{H} \alpha$ emission. It is a weaker detection than in the south with an $S / N \sim 3.4$ and an integrated $S / N \sim 4.6$ (see Fig. 1, right, top panel). The line is centered at a velocity about $50 \mathrm{~km} \mathrm{~s}^{-1}$ larger than the southern line, and its FWHM is smaller by a factor of two. The parameters of a Gaussian fit are given in Table 1. The corresponding mean $\mathrm{H}_{2}$ column density and $\mathrm{H}_{2}$ mass are $\sim 0.6 M_{\odot} \mathrm{pc}^{-2}$ and $\sim 0.8 \times 10^{8} M_{\odot}$, respectively.

There is no significant line detection in $\mathrm{CO}(2-1)$ in either region, although a weak signal at velocities around the $\mathrm{CO}(1-0)$ line suggests a marginal detection in the southern region (dashed-line spectra in Fig. 1, right panels). The rms sensitivity of the $\operatorname{CO}(2-1)$ spectrum per $52 \mathrm{~km} \mathrm{~s}^{-1}$ channel is $0.86 \mathrm{mK}$ and $1.1 \mathrm{mK}$ in the southern and northern regions, respectively. Using the widths of the $\mathrm{CO}(1-0)$ line, we can estimate the $3 \sigma$ upper limit of the $\mathrm{CO}(2-1)$ luminosity. The corresponding upper limit on the $L_{\mathrm{CO}(2-1)}^{\prime} / L_{\mathrm{CO}(1-0)}^{\prime}$ line ratio in the southern region is $\sim 0.22$. When the size of the $\mathrm{CO}(1-0)$ beam is accounted for, which is four-times larger than the $\mathrm{CO}(2-1)$ beam, this value may be consistent with typical values of $\sim 0.8$ in normal starforming galaxies (Saintonge et al. 2017; Leroy et al. 2009). The lower value could also indicate that the molecular gas is diffuse, warmer, and weak in $\mathrm{CO}$ emission, due to heating by the neighboring hot gas components (Peñaloza et al. 2017). In the northern region, most of the $\mathrm{CO}$ emission is probably not coming from the center of the beam, but is more widespread within the $\mathrm{CO}(1-0)$ beam.

A Gaussian fit to the southern $\mathrm{CO}(1-0)$ line leads to a velocity dispersion of $\sim 74 \mathrm{~km} \mathrm{~s}^{-1}$. Such a high value points to multiple molecular clouds with typical velocity dispersion less than a few $\mathrm{km} \mathrm{s}^{-1}$, but it may also be due to a velocity gradient in the beam. The $\mathrm{CO}$ velocity dispersion mirrors that of the median value of $\sim 60 \mathrm{~km} \mathrm{~s}^{-1}$ of the $\mathrm{H} \alpha$ emission. The velocity dispersion of the northern $\mathrm{CO}(1-0)$ emission is $\sim 37 \mathrm{~km} \mathrm{~s}^{-1}$, which may suggest that fewer molecular clouds were covered by the beam. The mean $\mathrm{H} \alpha$ velocity dispersion in the northern region is $\sim 52 \mathrm{~km} \mathrm{~s}^{-1}$. Higher resolution CO observations, resolving the emission in these areas, will be required to reveal the detailed morphology and kinematics of $\mathrm{CO}$ associated with the OC.

\section{Discussion}

\subsection{Correlation of gas phases}

The detected $\mathrm{CO}$ emission has velocities redshifted relative to the $\mathrm{H} \alpha$ component. Moreover, in the northern region, the $\mathrm{CO}$ emission is shifted to higher velocities relative to the southern region, while the underlying $\mathrm{H} \alpha$ emission in the two regions shows the opposite behavior.

The brightest $\mathrm{H} \alpha$ regions from MUSE (Ge et al. 2021) covered by the southern $\mathrm{CO}(1-0)$ beam have a median velocity of $c z \sim 7223 \mathrm{~km} \mathrm{~s}^{-1}$, while the central velocity of the $\mathrm{CO}(1-0)$ line is redshifted by $\sim 45 \mathrm{~km} \mathrm{~s}^{-1}$. The relatively large width of the $\mathrm{CO}$ line encompasses the $\mathrm{H} \alpha$ velocities. In the northern region, the only $\mathrm{H} \alpha$ emission is from the filaments at the edges of the $\mathrm{CO}(1-0)$ beam, with median velocities $c z \sim 7118-7230 \mathrm{~km} \mathrm{~s}^{-1}$ (with a representative value of $c z \sim 7182 \mathrm{~km} \mathrm{~s}^{-1}$ ), thus mostly blueshifted relative to the southern region. However, the northern CO line peaks $\sim 133 \mathrm{~km} \mathrm{~s}^{-1}$ higher and is thus rather strongly redshifted, both relative to the northern $\mathrm{H} \alpha$ emission as well as the southern CO line.

The velocity difference indicates that in the northern region, the cold and warm components are kinematically separated. In the southern region, the $\mathrm{CO}$ emission is more kinematically linked to the $\mathrm{H} \alpha$ emission, possibly due to a richer distribution of gas that covers a wider range in velocities.

The correlation of cold molecular and warm ionized phases is further explored in Fig. 2, where the two regions are plotted on the $\mathrm{CO}-\mathrm{H} \alpha$ correlation suggested from previous observations 
of RPS tails (Jáchym et al. 2017). In the southern OC region, the integrated $\mathrm{CO}$ intensity and the $\mathrm{H} \alpha$ surface brightness enclosed within the IRAM beam are consistent with the correlation. The northern region is slightly offset - it has a factor of $\sim 2$ lower $\mathrm{H} \alpha$ surface brightness for its $\mathrm{CO}$ integrated flux density. The plot in Fig. 2 comprises tail regions with a predominantly diffuse warm ionized gas component and no or only a few compact HII regions (the highest contribution of HII regions is in the inner part of the tail of the Norma galaxy ESO 137-001 - point 001A in the plot, where it makes up about $37 \%$ of the $\mathrm{H} \alpha$ flux). The correlation was measured at spatial scales of $\sim 5-10 \mathrm{kpc}$.

In addition, Ge et al. (2021) examined the correlation between the warm and hot ionized phases in the OC and compared it with the tight $\mathrm{H} \alpha-\mathrm{X}$-ray correlation recently found for RPS tails (Sun et al. 2021). They found excellent agreement for the southern region, while the $\mathrm{X}-\mathrm{ray} / \mathrm{H} \alpha$ ratio in the region encompassing our northern region is higher by a factor of $\sim 4$ (Ge et al. 2021, see their Fig. 8).

Thus, the conditions in the southern region covering the main $\mathrm{H} \alpha$ cloud seem to be close to the stripped gas in typical RPS tails, where tight $\mathrm{H} \alpha-\mathrm{CO}$ and $\mathrm{H} \alpha-\mathrm{X}$-ray correlations are observed. In the northern region, on the other hand, the gas may be locally in a different evolutionary phase with the elevated $\mathrm{X}$-ray/ $\mathrm{H} \alpha$ and $\mathrm{CO} / \mathrm{H} \alpha$ ratios. In the north, the gas phases are physically separated: the peak of X-ray emission is surrounded by the colder $\mathrm{H} \alpha$ emission filaments, from which the $\mathrm{CO}$ emission is kinematically offset. It may be expected that the cold gas is also spatially offset from the hot gas, given the surprising fact that there is a cold gas associated with the location of the X-ray peak.

Spatial and kinematic offsets are characteristic for RPS tails, where different gas components may be both spatially and kinematically offset due to the effects of differential acceleration by ram pressure. For example, offsets of the $\mathrm{CO}, \mathrm{H} \alpha$, and $\mathrm{X}$-ray emission were observed in the tail of the Norma cluster galaxy ESO 137-001 (Jáchym et al. 2019). In the Coma cluster galaxy D100, a kinematical offset of $\sim 50 \mathrm{~km} \mathrm{~s}^{-1}$ was measured along the tail between the $\mathrm{H} \alpha$ - and $\mathrm{CO}$-emitting components (Jáchym et al. 2017). Future detailed CO mapping will show whether cold and warm gas velocity gradients occur along a consistent direction, and how the $\mathrm{X}-\mathrm{ray}-\mathrm{H} \alpha$ offset relates to it.

\subsection{Mass budget}

Following the estimates of Ge et al. (2021), the mass budget of the part of the OC covered by the MUSE observations is as follows: there is about $8 \times 10^{7} M_{\odot}$ of warm $\mathrm{H} \alpha$ gas and about $3 \times 10^{9} M_{\odot}$ of hot X-ray gas (which is about a third of the total OC X-ray emission). With the $\sim 2.2 \times 10^{8} M_{\odot}$ of molecular gas detected in the two regions, our observations indicate that a molecular component may form about $10 \%$ of the total mass of the cloud, and that there is more cold than warm gas. From VLA observations (Scott et al. 2018), an upper limit on HI content in the area covering the cloud can be calculated (from their field $\mathrm{D}$ observed in the velocity range of $7091-7463 \mathrm{~km} \mathrm{~s}^{-1}$ ). For the OC region in the primary beam-corrected cube smoothed spatially to $45^{\prime \prime}$, which is close to the size of the OC, the measured rms is $\sim 0.67 \mathrm{mJy}$ in $11 \mathrm{~km} \mathrm{~s}^{-1}$ channels. Assuming the linewidths of the CO lines of 90 and $180 \mathrm{~km} \mathrm{~s}^{-1}$, the corresponding $\mathrm{HI}$ upper limits (corresponding to a $3 \sigma$-significance across three channels) are $\sim 2.2 \times 10^{8} M_{\odot}$ and $\sim 3.1 \times 10^{8} M_{\odot}$, respectively, thus similar to the lower limit on the molecular gas content.

\subsection{Inefficient star formation}

Analyses by Yagi et al. (2017) and Ge et al. (2021) indicated that star formation is almost absent in the OC. The upper limit for the star formation rate (SFR) of $6 \times 10^{-4} M_{\odot} \mathrm{yr}^{-1}$ was estimated from the lack of any GALEX sources in the MUSE field, with the calibration from Kennicutt \& Evans (2012). The only HII candidate in the MUSE field was identified outside the main $\mathrm{H} \alpha$ cloud at RA 11:44:21.4, Dec +20:10:14.6 (see Fig. 3 in Ge et al. 2021).

For the typical molecular gas depletion time in nearby spiral galaxies $\tau_{\mathrm{dep}, \mathrm{H}_{2}} \approx 2 \mathrm{Gyr}$ (e.g., Bigiel et al. 2011; Leroy et al. 2013), the molecular gas mass of $2.2 \times 10^{8} M_{\odot}$ would correspond to a $\mathrm{SFR}=M_{\mathrm{H}_{2}} / \tau_{\mathrm{dep}, \mathrm{H}_{2}} \sim 0.1 M_{\odot} \mathrm{yr}^{-1}$. The observed upper limit on the SFR is thus more than two orders of magnitude lower, indicating that the process of converting molecular gas into stars in the OC is extremely inefficient ${ }^{2}$. The inefficiency further increases when the atomic HI content's upper limit is taken into account.

The detected molecular gas is in a non-star-forming state. The physical conditions in the OC are likely distinct from galactic disks where star formation typically occurs. Some factors which, under typical conditions, drive and regulate star formation might be absent in the OC (e.g., the gravitational potential of a galaxy disk, stellar feedback, and UV background radiation), while some others are likely present (e.g., heat conduction or shocks from the surrounding ICM). This is similar to the conditions in RPS tails, where star formation can occur when it is still close to the galaxy, or just recently stripped, but it becomes less likely with increasing time and distance.

The $\mathrm{CO}$ velocity dispersion in the $\mathrm{OC}$ is rather high and similar to the velocity dispersion in the warm gas, which makes the gas less likely to be self-gravitating. While a highly resolved observations of the velocity field would be needed, shocks induced by ram pressure may increase the turbulent kinetic energy which stabilize molecular clouds, increasing their virial parameter and preventing them from collapse (e.g., Sivanandam et al. 2010).

The density of the molecular gas may play an important role in the low star-formation efficiency. The low mean surface density of $\sim 1 M_{\odot} \mathrm{pc}^{-2}$ corresponding to the measured integrated $\mathrm{CO}$ intensity suggests that the volume density of the molecular gas is very low, or that the fraction of the dense molecular phase that is more tightly correlated with star formation (e.g., Gao \& Solomon 2004) in the OC is limited. Recent observations of RPS tails indicated that an important fraction of the molecular component is extended, and its fraction increases with the distance from the parent galaxy (Jáchym et al. 2019; Moretti et al. 2020).

For comparison, the known examples of jellyfish tails with a detected molecular component revealed molecular gas depletion times $>10^{10} \mathrm{yr}$ (Jáchym et al. 2014, 2017; Moretti et al. $2018,2020)$. Their star formation rates vary in the range of $\sim 0.01-1 M_{\odot} \mathrm{yr}^{-1}$ (e.g., Poggianti et al. 2019; Cramer et al. 2019; Kenney et al. 2014). One of the extreme cases is the tail of the Coma cluster RPS galaxy D100, where for the detected $M_{\mathrm{H}_{2}} \sim 10^{9} M_{\odot}$, the SFR is only $\sim 6.0 \times 10^{-3} M_{\odot} \mathrm{yr}^{-1}$, and thus $\tau_{\mathrm{dep}, \mathrm{H}_{2}} \sim 1.6 \times 10^{11} \mathrm{yr}$. Long depletion times were previously observed locally in some tidally disturbed galaxies (e.g., Tomičić et al. 2018), gas disks in early-type galaxies that are more stable against fragmentation to dense clumps

\footnotetext{
2 We note that uncertainty in the CO-to- $\mathrm{H}_{2}$ conversion factor cannot account for such a low efficiency and extremely long depletion times.
} 
due to the lower disk self-gravity and increased shear (e.g., Martig et al. 2013), HI-excess galaxies in low-density environments (Geréb et al. 2018), or outer parts of spiral disks which, however, are dominated by a (low column density) HI component (Bigiel et al. 2010; Y1ld1z et al. 2017).

\subsection{Origin of the molecular OC}

Given the large projected distance of $>80 \mathrm{kpc}$ of the OC from any possible parent galaxy, it is reasonable to suggest that the $\mathrm{OC}$ is in an advanced evolutionary state and that the original cold gas was heated due to mixing with the hot ICM. In RPS tails, compact molecular gas detected at large (several tens of kiloparsecs) distances from the main galaxies are expected to have formed in situ from the stripped gas (Jáchym et al. 2019; Moretti et al. 2020).

Dust survival is an essential element of the formation of molecular gas (e.g., Hollenbach \& McKee 1979). This implies that in the OC, the stripped gas that has mixed with the ICM wind still keeps a fraction of its initial dust content. Recent $3 \mathrm{D}$ hydrodynamic simulations of the evolution of cold $\left(10^{3} \mathrm{~K}\right)$ dusty clouds in a hot wind indicate that if the characteristic cooling timescales of the mixed gas are shorter than the cloud crushing time, the atomic phase can survive, and once the cloud is entrained by the wind, it will cool down and molecular gas may reform (Farber \& Gronke 2022; Gronke \& Oh 2020; Kanjilal et al. 2021). Another factor that could help the recent formation of molecular gas is compression due to ram pressure. The $\mathrm{H} \alpha$ OC has a velocity gradient nearly aligned in the eastwest direction (Ge et al. 2021), which, together with the elevated values of the $\mathrm{H} \alpha$ velocity dispersion at the west side of the OC, may suggest that the west edge experiences an ICM ram pressure.

\subsection{Molecular content of a nearby RPS galaxy}

About $100 \mathrm{kpc}$ southward of the OC at the same redshift lies the galaxy 2MASX J11443212+2006238, which has an $85 \mathrm{kpc}$ long $\mathrm{H} \alpha$ ram pressure stripped tail (Gavazzi et al. 2017, see Fig. 1, left panel). Both $\mathrm{CO}(1-0)$ and $\mathrm{CO}(2-1)$ emission was strongly detected in the main body of the galaxy (see the inset spectra in Fig. 1, left panel), with a corresponding $\mathrm{H}_{2}$ mass of $\sim 2.4 \times 10^{9} M_{\odot}$. Table 1 summarizes the rms sensitivity of the $\mathrm{CO}$ data in $20 \mathrm{~km} \mathrm{~s}^{-1}$ channels and the parameters of the Gaussian fits. The fits indicate that the line profiles are slightly asymmetric, especially in $\mathrm{CO}(1-0)$, likely corresponding to the redistribution of the molecular gas due to ram pressure stripping. The $\mathrm{CO}$ emission shows no offset in radial velocity from $\mathrm{H} \alpha$ (Gavazzi et al. 2017).

2MASX J11443212+2006238 illustrates an early phase of ram pressure stripping when the tail is connected to a starforming, $\mathrm{H} \alpha$-bright disk (Yagi et al. 2017). This is in obvious contrast to the late evolutionary phase of the neighboring OC which has detached from its parent galaxy and has evolved and mixed with the hot ICM.

Despite the similar velocities and the projected proximity, there is no direct observational evidence that the galaxy is related to the OC; also, 2MASX could not be the RPS parent of the OC either since its radial velocity would likely need to be substantially larger (redshifted) than that of the OC. While the origin of the OC will be studied elsewhere, we note that there are several galaxy pairs with a known HI-deficient late-type galaxy member near the OC (Scott et al. 2010, 2018). A pair tidal interaction loosening the ISM from the pair's gravitational potential with subsequent RPS could also be a viable scenario for the origin of the gas in the OC.

\section{Conclusions}

Using the IRAM 30m telescope, we have detected $\operatorname{CO}(1-0)$ emission in two regions of the multiphase OC. This recently discovered object lies at $\sim 800 \mathrm{kpc}$ north of the center of the Abell 1367 galaxy cluster. About $1.4 \times 10^{8} M_{\odot}$ of $\mathrm{H}_{2}$ was detected in the region covering the peak in $\mathrm{H} \alpha$ emission (assuming a standard Galactic CO-to- $\mathrm{H}_{2}$ conversion factor) and about $8 \times 10^{7} M_{\odot}$ in the region separated by $\sim 12 \mathrm{kpc}$, where X-ray emission has a peak, surrounded by $\mathrm{H} \alpha$ filaments, as shown by the MUSE observations (see Fig. 1; Ge et al. 2021).

In the region covering the $\mathrm{H} \alpha$ peak, the conditions seem to be consistent with that of gas in typical ram pressure stripped tails and follows the established tight $\mathrm{H} \alpha-\mathrm{X}$-ray and $\mathrm{H} \alpha-\mathrm{CO}$ correlations. In the region covering the X-ray peak, the X-ray/H $\alpha$ and $\mathrm{CO} / \mathrm{H} \alpha$ ratios are elevated by factors of 4 and 2, respectively, and the mean velocity of the $\mathrm{CO}$ emission is offset from the warm ionized component by more than $100 \mathrm{~km} \mathrm{~s}^{-1}$. This may support the scenario of the RPS origin of the OC since spatial and kinematics offsets of gas phases (as well as the presence of a velocity gradient) are characteristic of RPS tails.

The $\mathrm{CO}$ velocity dispersion in the two regions is rather high $\left(\sim 70 \& 40 \mathrm{~km} \mathrm{~s}^{-1}\right)$. Together with the presumably low molecular gas volume density, this may be the principal factor in the extremely low star formation efficiency of the detected molecular gas $\left(\tau_{\text {dep, } \mathrm{H}_{2}} \sim 3.7 \times 10^{11} \mathrm{yr}\right)$.

The OC likely represents a late evolutionary product of a gas stripping event of an unidentified cluster galaxy. Its total mass is dominated by the hot ionized component, while the molecular gas forms about $10 \%$ of the total mass budget, similar to the upper limit contribution by $\mathrm{HI}$ emission calculated from existing VLA observations.

In contrast to the aged OC, we also observed the nearby galaxy 2MASX J11443212+2006238, which is in an early stage of ram pressure stripping, and detected $\sim 2.4 \times 10^{9} M_{\odot}$ of molecular gas in its main body.

Acknowledgements. We warmly thank the IRAM 30-m observatory staff for their help during the observations and with the preparation of observing scripts. P. J. acknowledges support from the project LM2018106 of the Ministry of Education, Youth and Sports of the Czech Republic and from the project RVO:67985815. M.S. acknowledges support provided by the NASA grant 80 NSSC19K0953 and the NSF grant 1714764. T. S. acknowledges support by Fundação para a Ciência e a Tecnologia (FCT) through national funds (UID/FIS/04434/2013), FCT/MCTES through national funds (PIDDAC) by this grant UID/FIS/04434/2019 and by FEDER through COMPETE2020 (POCI-01-0145-FEDER-007672). T. S. also acknowledges support from DL 57/2016/CP1364/CT0009.

\section{References}

Beccari, G., Bellazzini, M., Magrini, L., et al. 2017, MNRAS, 465, 2189 Bigiel, F., Leroy, A., Walter, F., et al. 2010, AJ, 140, 1194

Bigiel, F., Leroy, A. K., Walter, F., et al. 2011, ApJ, 730, L13 Boselli, A., \& Gavazzi, G. 2006, PASP, 118, 517

Cramer, W. J., Kenney, J. D. P., Sun, M., et al. 2019, ApJ, 870, 63

Davies, J., Minchin, R., Sabatini, S., et al. 2004, MNRAS, 349, 922

Farber, R. J., \& Gronke, M. 2022, MNRAS, 510, 551

Fossati, M., Fumagalli, M., Gavazzi, G., et al. 2019, MNRAS, 484, 2212

Gao, Y., \& Solomon, P. M. 2004, ApJ, 606, 271

Gavazzi, G., Boselli, A., Mayer, L., et al. 2001, ApJ, 563, L23

Gavazzi, G., Cortese, L., Boselli, A., et al. 2003, ApJ, 597, 210

Gavazzi, G., Consolandi, G., Yagi, M., et al. 2017, A\&A, 606, A131

Ge, C., Sun, M., Liu, R.-Y., et al. 2019, MNRAS, 486, L36 
A\&A 658, L5 (2022)

Ge, C., Luo, R., Sun, M., et al. 2021, MNRAS, 505, 4702

Geréb, K., Janowiecki, S., Catinella, B., et al. 2018, MNRAS, 476, 896

Gronke, M., \& Oh, S. P. 2020, MNRAS, 492, 1970

Hollenbach, D., \& McKee, C. F. 1979, ApJS, 41, 555

Iglesias-Páramo, J., Boselli, A., Cortese, L., et al. 2002, A\&A, 384, 383

Jáchym, P., Combes, F., Cortese, L., et al. 2014, ApJ, 792, 11

Jáchym, P., Sun, M., Kenney, J. D. P., et al. 2017, ApJ, 839, 114

Jáchym, P., Kenney, J. D. P., Sun, M., et al. 2019, ApJ, 883, 145

Kanjilal, V., Dutta, A., \& Sharma, P. 2021, MNRAS, 501, 1143

Kenney, J. D. P., Geha, M., Jáchym, P., et al. 2014, ApJ, 780, 119

Kennicutt, R. C., \& Evans, N. J. 2012, ARA\&A, 50, 531

Kent, B. R., Giovanelli, R., Haynes, M. P., et al. 2007, ApJ, 665, L15

Leroy, A. K., Walter, F., Bigiel, F., et al. 2009, AJ, 137, 4670

Leroy, A. K., Walter, F., Sandstrom, K., et al. 2013, AJ, 146, 19

Martig, M., Crocker, A. F., Bournaud, F., et al. 2013, MNRAS, 432, 1914
Moretti, A., Paladino, R., Poggianti, B. M., et al. 2018, MNRAS, 480, 2508 Moretti, A., Paladino, R., Poggianti, B. M., et al. 2020, ApJ, 889, 9

Peñaloza, C. H., Clark, P. C., Glover, S. C. O., et al. 2017, MNRAS, 465, 2277 Poggianti, B. M., Gullieuszik, M., Tonnesen, S., et al. 2019, MNRAS, 482, 4466 Saintonge, A., Catinella, B., Tacconi, L. J., et al. 2017, ApJS, 233, 22

Scott, T. C., Bravo-Alfaro, H., Brinks, E., et al. 2010, MNRAS, 403, 1175

Scott, T. C., Brinks, E., Cortese, L., et al. 2018, MNRAS, 475, 4648

Sivanandam, S., Rieke, M. J., \& Rieke, G. H. 2010, ApJ, 717, 147

Solomon, P. M., \& Vanden Bout, P. A. 2005, ARA\&A, 43, 677

Sun, M., Donahue, M., Roediger, E., et al. 2010, ApJ, 708, 946

Sun, M., Ge, C., Luo, R., et al. 2021, Nat. Astron., in press [arXiv:2103.09205] Tomičić, N., Hughes, A., Kreckel, K., et al. 2018, ApJ, 869, L38

Wong, O. I., Stevens, A. R. H., For, B.-Q., et al. 2021, MNRAS, 507, 2905

Yagi, M., Yoshida, M., Gavazzi, G., et al. 2017, ApJ, 839, 65

Yıldız, M. K., Serra, P., Peletier, R. F., et al. 2017, MNRAS, 464, 329 